\title{
Correlation between tuberculin skin test and IGRAs with risk factors for the spread of infection in close contacts with sputum smear positive in pulmonary tuberculosis
}

\author{
Maria Luiza de Souza-Galvão 1,2,3*, Irene Latorre ${ }^{3,4,6}$, Neus Altet-Gómez ${ }^{1,2}$, María Ángeles Jiménez-Fuentes ${ }^{1}$, \\ Celia Milà', Jordi Solsona', Maria Asunción Seminario ${ }^{1}$, Adela Cantos ${ }^{1}$, Juan Ruiz-Manzano ${ }^{3,5,6}$ \\ and José Domínguez $3,4,6$
}

\begin{abstract}
Background: The aim of the study was to assess the correlation between the tuberculin skin test (TST) and in vitro interferon-gamma released assays (IGRAs) with risk factors for the spread of infection in smear positive pulmonary tuberculosis (TB) contacts.

Methods: We recruited prospective contacts with smear positive pulmonary TB cases. We looked at human immunodeficiency virus (HIV) infection and other conditions of immunosuppression, presence of BCG vaccination and the degree of exposure to the index case. Patients underwent the TST, chest radiography, sputum analysis when necessary, and IGRA assays (QFN-G-IT and T-SPOT.TB). Presence of cough, diagnostic delay (days between first symptoms and TB diagnostic), contact conditions: room size (square meters) and index of overcrowding (square meters per person) were investigated in the index case.
\end{abstract}

Results: 156 contacts (119 adults, 37 children) of 66 TB patients were enrolled, 2.4 (1-14) contacts per TB case. The positivity of the TST did not correlate with the risk factors studied: presence of cough $(p=0.929)$; delayed diagnosis $(p=0.244)$; room size $(p=0.462)$; overcrowding $(p=0.800)$. Both QFN-G-IT and T-SPOT.TB, showed significant association with cough ( $p=0.001$, and $p=0.007)$ and room size $(p=0.020$, and $p=0.023)$, respectively.

Conclusions: Both IGRA associated better than TST with certain host-related risk factors involved in the transmission of disease, such as the presence of cough.

Keywords: Tuberculosis infection, Tuberculin skin test, Interferon gamma release assays, IGRA, Overcrowding, Diagnostic delay, Cough

\section{Background}

The World Health Organization estimates approximately 8.6 million new cases of tuberculosis (TB) annually [1]. Studies published in the 80 s showed that $5-10 \%$ of recently infected contacts develop active disease within the subsequent 2-5 years after exposure to an infectious source; while another 5-10\% percent develop TB sometime

\footnotetext{
* Correspondence: msouza@vhebron.net

'Unitat de Tuberculosi de Drassanes, Hospital Universitari Vall d'Hebron, Barcelona, Spain

${ }^{2}$ IDIAP Jordi Gol Research Foundation, Barcelona, Spain

Full list of author information is available at the end of the article
}

during the rest of their lives [2]. In Barcelona, a low TB burden city (incidence: $24.9 / 100,000$ in 2011), a study showed that $25.5 \%$ of new TB cases were due to recent transmission [3].

TB prevention relies on the targeted identification and preventive treatment of individuals who carry an increased risk of developing TB [4]. Identification of individuals from these groups, showing the highest risk of developing TB is performed by the evaluation of the presence of an adaptive immunity against Mycobacterium tuberculosis, in the absence of active disease. For many decades the tuberculin skin test (TST) has been the immunodiagnostic method of 
choice for this risk analysis. In the last years, interferon- $\gamma$ (IFN- $\gamma$ ) release assays (IGRAs), the Quantiferon-TB Gold in Tube (QFN-G-IT) (QIAGEN, Düsseldorf, Germany), and the T-SPOT.TB (Oxford Immunotec Limited, Abingdon, UK), have been introduced into clinical practice as laboratory methods for the immunediagnosis of latent TB infection (LTBI) [5].

The spread of TB in confined spaces is strongly influenced by the number of infected droplet nuclei present in the air, the viability of the $M$. tuberculosis bacilli and the duration of exposure [6]. Living in a crowded household increases the risk of TB infection [7]. The presence of cough decreases the delay in TB diagnostic, improving suspicion, also increasing the spread of the disease [8]. While several studies have compared the frequency of positive TST and IGRA results in relation to the exposure time to case index $[9,10]$, no study comparing the performance of the TST and IGRAs was targeted directly in living conditions and on overcrowding, as risk factors for the development of LTBI in contacts.

The aim of this study was to assess the association between the positivity of TST and IGRAs with some risk factors for the spread of the disease as: presence of a cough and diagnostic delay in the index case; and the size of the room and the overcrowding in the environment where the contact took place. Furthermore, we compared the performance of the 3 tests, the agreement between them as well as their correlation with the time of exposure.

\section{Methods}

\section{Study design}

We prospectively recruited people who came to the TB Unit in Drassanes (Barcelona, Spain) in our routine contact investigation tasks. The Ethics Committee of the "IDIAP Jordi Gol Research Foundation" provided ethics approval for this study. Patients and parents of children were asked to sign a consent form. Detailed questionnaires were completed with data about prior contact or any prior TST result; data about HIV infection or other causes of immunosuppression were collected and we looked for the presence of BCG scars. We tested using TST and whenever possible, also with QFN-GIT and with T-SPOT.TB. We drew blood only from untreated patients or from patients receiving LTBI treatment for less than 30 days, in order to avoid the effects of the therapy skewing IGRA results [11]. Chest $x$-Ray and sputum analysis were also done if necessary.

Contacts were eligible if they were exposed to a pulmonary TB sputum smear positive patient index, and excluded if they had active TB, were diagnosed with HIV infection, were pregnant, or if the result of the culture of the patient index was not confirmed to be $M$. tuberculosis. Contacts of sputum smear positive patients were from two different origins: familial (relatives or friends referred to the Unit for TB screening) and community (colleagues or students referred to the Unit for screening with chest $\mathrm{x}$-Ray due to a positive TST). They were also classified according to degree of exposure to the case index in 2 groups: 6 or more hours daily and less than 6 hours daily. Clinicians were blind to IGRA results and microbiologists did not know the clinical history of the patients.

\section{Clinical and environmental risk factors for TB infection}

In the index case we checked for the presence or absence of a cough and considered the days of diagnostic delay, defined as days between the first symptoms and TB diagnostic. As far as the environmental contact conditions, we looked for data on the room size (in square meters) and we used the Floor Area per Person Index. The room size was classified according to the Statistical Institute of Catalonia, slightly adapted to the characteristics of the neighbourhood of our study population. This indicator of Sustainable Development was used by the United Nations, to measure the adequacy of living space, not taking into account cultural differences, as an indicator of overcrowding. We considered overcrowding when the index was less than 15 square meters per person [12]. The data about the index case were obtained by direct interview when the patient was diagnosed in our institution or collected from the database of the TB Programs of Barcelona and Catalonia.

\section{Tuberculin skin testing}

TST was performed by the Mantoux method using two tuberculin units of PPD RT23 (Statens Serum Institut, Copenhagen, Denmark) and read within 48-72 h, using the ball-point pen and ruler method by trained nurses and doctors. All TST $\geq 5 \mathrm{~mm}$ were classified as positive result regardless of the BCG defined by the Spanish Pneumology and Thoracic Surgery Society (SEPAR) guidelines [13,14].

\section{QFN-gold in tube}

One $\mathrm{ml}$ of blood was drawn per each of the 3 tubes with antigens ready for incubation: (Nil, TB antigens and mitogen). Samples were incubated with stimulatory antigens for $16 \mathrm{~h}-24 \mathrm{~h}$ and the test was performed according to the manufacturer's instructions. The cut-off value for a positive test was IFN- $\gamma$ of at least $0.35 \mathrm{IU} / \mathrm{mL}$ in the sample after stimulation with the specific antigens, regardless of the result of the mitogen control. The result of the test was considered indeterminate if the antigenstimulated sample was negative and if the value of the positive control was less than $0.5 \mathrm{IU} / \mathrm{mL}$ after subtraction of the value of the nil control, and/or if the nil control was higher than $8.0 \mathrm{IU} / \mathrm{ml}$. 


\section{T-SPOT.TB}

The test was performed following manufacturer's recommendations. $8 \mathrm{ml}$ of blood were drawn from each subject by venipuncture for the isolation of peripheral blood mononuclear cells (PBMCs) in CPT tube (Beckton Dickinson Diagnostics, Franklin Lakes, NJ). The isolated PBMCs were washed twice by centrifugation with RPMI medium (Invitrogen, Auckland, N.Z.), and later resuspended in AIM-V medium (Invitrogen, Auckland, N.Z.). Finally, viable cells were counted with an inverted microscope using the tripan blue method.

After that, the PBMCs were stimulated in each well by medium alone (as nil control), phytohaemagglutinin (as positive control), different peptide panels encompassing the antigens ESAT- 6 and CFP-10. Spots were scored using an automated AID ELISPOT plate reader (AID Systems, Strasberg, Germany) and also by naked eye. Test wells were scored as positive if they contained at least six spot-forming cells more than the nil control well, and if this number was at least twice the number of the nil control well. The result of the test was considered undetermined if the antigen-stimulated sample was negative and if the value of the positive control was less than 20 spots, and/or if the number of SFC in the negative control was greater than 10. All blood samples for both tests were processed within $4 \mathrm{~h}$ of phlebotomy in the Microbiology Department of the Hospital Universitari Germans Trias i Pujol in Badalona (Spain).

\section{Statistical analysis}

The qualitative variables description is based on the calculation of the number and its percentage, and for quantitative variables, based on calculation of the mean and the standard deviation (SD). The chi-squared test and two-tailed Fisher's exact test were used to compare qualitative variables. Non-parametric tests (Mann-Whitney, Kolmogorov-Smirnov, Kruskal-Wallis) were used to compare quantitative variables according to the categories of the group of variable. Cohen's kappa coefficient (k) was used to analyse the concordance and its $\mathrm{p}$ value. All analyses were made with SPSS statistical software for Windows (SPSS version 15.0; SPSS, Chigago, IL).

\section{Results}

\section{Demography}

Table 1 summarizes the demographic and clinical details. 230 contact patients with smear positive TB were recruited for the study. 11 patients were excluded by diagnosis of active TB; 5 by absence of growth of $M$. tuberculosis in the culture sputum of the index case and 34 because the blood samples were collected more than 30 days after they had started treatment of TB infection. 24 contacts did not have all three testing techniques applied. In the end, a total of 156 contacts (119 adults and 37 children) from 66 cases of
Table 1 Characteristics of the study population $(n=156)$

\begin{tabular}{|c|c|c|}
\hline Characteristics & Adults $n=119$ & Children $n=37$ \\
\hline Mean age (SD) & $29.3(10.5)$ & $9.2(2.4)$ \\
\hline \multicolumn{3}{|l|}{ Gender } \\
\hline Female & $67(56.3)$ & $21(56.8)$ \\
\hline Male & $52(43.7)$ & $16(43.2)$ \\
\hline \multicolumn{3}{|l|}{ BCG-vaccinated } \\
\hline Yes & $69(58)$ & $25(67.6)$ \\
\hline No & $50(42)$ & $12(32.4)$ \\
\hline \multicolumn{3}{|l|}{ Place of birth } \\
\hline Spain & $60(50.4)$ & $12(32.4)$ \\
\hline Outside Spain & $59(49.6)$ & $25(67.6)$ \\
\hline America & $42(71.2)$ & $19(76)$ \\
\hline Eastern Europe Mediterranean & $7(11.9)$ & $1(4)$ \\
\hline Europe & $6(5)$ & $5(13.5)$ \\
\hline South Asia & $1(1.7)$ & $0(0)$ \\
\hline Western Pacific & $3(5.1)$ & $0(0)$ \\
\hline \multicolumn{3}{|l|}{ Type of contact } \\
\hline Familial & $45(37.8)$ & $25(67.6)$ \\
\hline Community & $74(62.1)$ & $12(32.4)$ \\
\hline \multicolumn{3}{|l|}{ Degree of exposure } \\
\hline$\geq 6$ hours daily & $46(38.6)$ & $16(43.2)$ \\
\hline$<6$ hours daily & $73(61.4)$ & $21(56.8)$ \\
\hline \multicolumn{3}{|l|}{ Cough in index case } \\
\hline Yes & $37(52.1)$ & $23(92)$ \\
\hline No & $34(47.9)$ & $2(8)$ \\
\hline
\end{tabular}

Data are presented as $\mathrm{n}(\%)$ or means \pm SD. BCG: Bacille Calmette-Guérin.

pulmonary TB sputum smear positive were enrolled (Figure 1). The average number of close contacts per source case was 2.4 and varied from 1 to 14 individuals.

\section{Agreement between tests}

Overall agreement of TST and both IGRAs was mostly slight (Table 2). Nevertheless, in BCG-unvaccinated children reached the best agreement. The overall agreement between both IGRAs was substantial; with no differences in the agreement in BCG-vaccinated and nonBCG-vaccinated taking into account children and adults together.

\section{Positive test results for all techniques}

The results of TST and IGRAs are summarised separately into adults and children according with the origin of the contact (familial or community contact) in Figure 2, and also according to the presence or absence of $\mathrm{BCG}$ vaccine and the degree of exposure (Tables 3 and 4). 


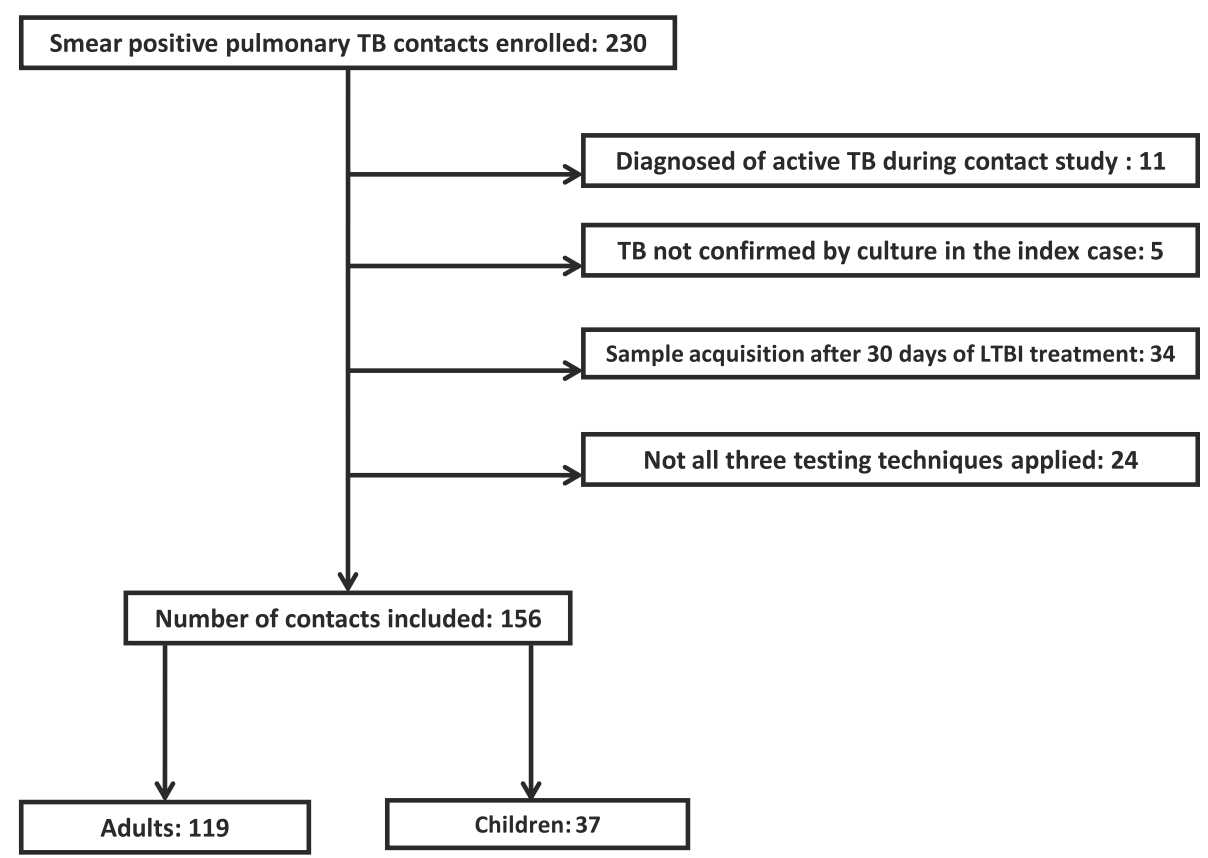

Figure 1 Patients included and causes for exclusion from the study (TB: tuberculosis. LTBI: latent tuberculosis infection).

\section{BCG-unvaccinated}

The results of the TST showed 46 (92\%) positive adults and 11 (91\%) positive children (Table 3). The QFN-G-IT was positive in $27(54 \%)$ adults and $9(75 \%)$ children, while the T-SPOT.TB was positive in $33(66 \%)$ and indeterminate in $2(4 \%)$ adults; and positive in $10(83.3 \%)$ children with no indeterminate results.

\section{BCG-vaccinated}

Percentages of positive results in TST were similar to those of non-vaccinated individuals $(92.8 \%$ and $92 \%$ in adults and children, respectively) (Table 4). In contrast, IGRAs detected less positive results compared to the unvaccinated groups, except for T-SPOT.TB in children.

\section{Positive test results according to degree of exposure BCG-unvaccinated contacts}

TST positive results did not correlate with the degree of exposure in adults, showing identical percentage of positive results independently of the degree exposure (Table 3). In contrast, although IGRAs showed a higher amount of positive results with the increase of hours of

Table 2 Agreement between tests and K coefficients

\begin{tabular}{|c|c|c|c|c|c|c|}
\hline \multirow{2}{*}{$\begin{array}{l}\text { Patient } \\
\text { characteristics }\end{array}$} & \multicolumn{2}{|c|}{ TST versus T-SPOT-TB } & \multicolumn{2}{|c|}{ TST versus QFN-IT } & \multicolumn{2}{|c|}{ T.SPOT-TB versus QFN-IT } \\
\hline & Agreement (\%) & $\overline{\text { Kappa }}$ & Agreement (\%) & Kappa & Agreement (\%) & Kappa \\
\hline \multicolumn{7}{|l|}{ Total } \\
\hline All & 62.6 & 0.172 & 50.91 & 0.121 & 82.82 & 0.659 \\
\hline BCG & 51.58 & 0.119 & 42 & 0.078 & 83.84 & 0.664 \\
\hline No BCG & 79.37 & 0.310 & 64.62 & 0.224 & 81.25 & 0.604 \\
\hline \multicolumn{7}{|l|}{ Adults } \\
\hline All & 58.97 & 0.148 & 45.6 & 0.100 & 81.45 & 0.629 \\
\hline BCG & 47.83 & 0.101 & 34.72 & 0.056 & 80.82 & 0.580 \\
\hline No BCG & 75 & 0.256 & 60.38 & 0.195 & 82.35 & 0.637 \\
\hline \multicolumn{7}{|l|}{ Children } \\
\hline All & 75 & 0.288 & 69.23 & 0.235 & 86.84 & 0.721 \\
\hline BCG & 64 & 0.199 & 62.96 & 0.182 & 92 & 0.838 \\
\hline No BCG & 93.33 & 0.634 & 83.33 & 0.429 & 76.92 & 0.418 \\
\hline
\end{tabular}

BCG: Bacille Calmette-Guérin. TST: tuberculin skin test. QFN-G-IT: Quantiferon-TB Gold in tube. 


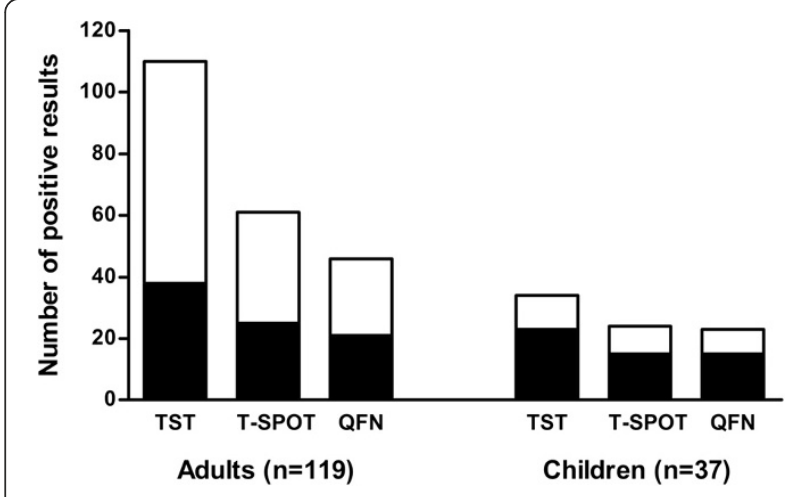

Figure 2 Number of positive results obtained by TST and both IGRA tests (T-SPOT.TB and QFN-G-IT) in adults and children, including information in each case of the origin of the contact: familial ( $\square$ ) or from the community ( $\square)$.

exposure to the index case, the differences did not reached statistical significance.

Taking into account the same degree of exposure, significant differences were found between the positive results using the 3 techniques. TST compared with QFN-G-IT and T-SPOT.TB showed more significant positive results in both degrees of exposure: more than 6 hours ( $\mathrm{p}=0.007$ and $\mathrm{p}=0.022$, respectively) and less than 6 hours $(\mathrm{p}<0.001$, respectively) in adults. The low number of BCG-unvaccinated children included, did not allow us to establish if there was a statistical association in this group.

\section{BCG-vaccinated contacts}

TST did not show a significant difference in the number of positive results according to the degree of exposure, neither in adults nor in children (Table 4). In opposite, although IGRAs showed a higher amount of positive results with the increase of hours of exposure to the index case, the differences did not reached statistical significance.
Taking into account the same degree of exposure, significant differences were found between the positive results using the 3 techniques. TST compared with QFN-G-IT and T-SPOT.TB showed more positive results in both degrees of exposure: more than 6 hours ( $\mathrm{p}<0.001$, respectively) and less than 6 hours ( $\mathrm{p}<$ 0.001 , respectively) in adults and the same in children ( $\mathrm{p}=0.018$ and 0.016 , respectively).

\section{Quantitative evaluation of T-cell response according to the degree of exposure}

We studied T-cell quantitative enumeration after stimulation with ESAT-6, CFP-10 and also the sum of both antigens together (ESAT6/CFP10) with T-SPOT.TB; and the amount of IFN- $\gamma$ release in QFT-G-IT, in the contacts included, according to the different degrees of exposure to the index case. In adults, we found a higher amount of responding $\mathrm{T}$ cells after stimulation with CFP-10 ( $\mathrm{p}=$ $0.029)$, ESAT6/CFP10 $(p=0.042)$ and in the amount of IFN- $\gamma$ released $(p=0.015)$ (Figure 3$)$ in contacts with $>6 \mathrm{~h}$ of exposure, in comparison to the contacts with $<6 \mathrm{~h}$ of exposure. In children, this analysis was not possible, given the low number of children included.

\section{Positive results according to clinical and environmental risk factors}

We studied 4 risk factors likely associated with TB infection: "presence of cough", "days of diagnostic delay", "size of the household contact" and "overcrowding", and their relation with the positivity of the TST, QFN-GIT and T-SPOT.TB. In the paediatric population, the positivity of the techniques was not correlated with any of the risk factors studied. Data concerning the adult population is presented in Table 5. For the TST, the positivity of the test was not correlated with any of the risk factors studied. On the other hand, we found that the positivity of both IGRAs tests were associated with presence of

Table 3 Results of interferon- $\gamma$ release assays and tuberculin skin test in adults and children BCG-unvaccinated

\begin{tabular}{|c|c|c|c|c|c|c|c|c|c|}
\hline \multirow{3}{*}{ Technique } & & \multicolumn{4}{|c|}{ Adults } & \multicolumn{4}{|c|}{ Children } \\
\hline & & \multicolumn{4}{|c|}{ Degree of exposure } & \multicolumn{4}{|c|}{ Degree of exposure } \\
\hline & & $>6 \mathrm{~h}$ daily & $<6$ h daily & Total & $p$ & $>6$ h daily & $<6$ h daily & Total & $p$ \\
\hline \multirow[t]{2}{*}{ TST } & Positive & $18(94.7)$ & $28(90.3)$ & $46(92)$ & 0.820 & $2(100)$ & $9(90)$ & $11(91)$ & 0.999 \\
\hline & Negative & $1(5.3)$ & $3(9.7)$ & $4(8)$ & & $0(0)$ & $1(10)$ & $1(9)$ & \\
\hline \multirow[t]{3}{*}{ QFN-G-IT } & Positive & $13(68.4)$ & $14(45.2)$ & $27(54)$ & 0.173 & $2(100)$ & $7(70)$ & $9(75)$ & 0.999 \\
\hline & Negative & $6(31.6)$ & $17(54.8)$ & $23(46)$ & & $0(0)$ & $3(30)$ & $3(25)$ & \\
\hline & Indeterminate & $0(0)$ & $0(0)$ & $0(0)$ & & $0(0)$ & $0(0)$ & $0(0)$ & \\
\hline \multirow[t]{3}{*}{ T.SPOT-TB } & Positive & $15(78.9)$ & $18(58)$ & $33(66)$ & 0.223 & $2(100)$ & $8(80)$ & $10(83.3)$ & 0.999 \\
\hline & Negative & $4(21.1)$ & $11(35.5)$ & $15(30)$ & & $0(0)$ & $2(20)$ & $2(16.7)$ & \\
\hline & Indeterminate & $0(0)$ & $2(6.5)$ & $2(4)$ & & $0(0)$ & $0(0)$ & $0(0)$ & \\
\hline
\end{tabular}

Data are presented as n (\%). BCG: Bacille Calmette-Guérin. TST: tuberculin skin test. QFN-G-IT: Quantiferon-TB Gold in tube.

Two-tailed statistical test was used to compare the qualitative results of TST, QFN-G-IT and T-SPOT.TB, respectively, between the two degree of exposure categories in adults and children. 
Table 4 Results of interferon- $\gamma$ release assays and tuberculin skin test in adults and children BCG-vaccinated

\begin{tabular}{|c|c|c|c|c|c|c|c|c|c|}
\hline \multirow{3}{*}{ Technique } & & \multicolumn{4}{|c|}{ Adults } & \multicolumn{4}{|c|}{ Children } \\
\hline & & \multicolumn{4}{|c|}{ Degree of exposure } & \multicolumn{4}{|c|}{ Degree of exposure } \\
\hline & & $>6 \mathrm{~h}$ daily & $<6 \mathrm{~h}$ daily & Total & $\mathrm{p}$ & $>6 \mathrm{~h}$ daily & $<6$ h daily & Total & $\mathrm{p}$ \\
\hline \multirow[t]{2}{*}{ TST } & Positive & $27(100)$ & $37(88.1)$ & $64(92.7)$ & 0.998 & $13(92.9)$ & $10(90.9)$ & $23(92)$ & 0.859 \\
\hline & Negative & $0(0)$ & $5(11.9)$ & $5(7.3)$ & & $1(7.1)$ & $1(9.1)$ & $2(8)$ & \\
\hline \multirow[t]{3}{*}{ QFN-G-IT } & Positive & $9(33.3)$ & $10(23.8)$ & $19(27.5)$ & 0.622 & $9(64.3)$ & $5(45.5)$ & $14(56)$ & 0.350 \\
\hline & Negative & $17(63)$ & $32(76.2)$ & $49(71)$ & & $5(35.7)$ & $6(54.5)$ & $11(44)$ & \\
\hline & Indeterminate & $1(3.7)$ & $0(0)$ & $1(1.5)$ & & $0(0)$ & $0(0)$ & $0(0)$ & \\
\hline \multirow[t]{3}{*}{ T.SPOT-TB } & Positive & $14(51.8)$ & $14(33.3)$ & $28(40.6)$ & 0.175 & $9(64.3)$ & $5(45.5)$ & $14(56)$ & 0.350 \\
\hline & Negative & $13(48.2)$ & $28(66.7)$ & $41(59.4)$ & & $5(35.7)$ & $6(54.5)$ & $11(44)$ & \\
\hline & Indeterminate & $0(0)$ & $0(0)$ & $0(0)$ & & $0(0)$ & $0(0)$ & $0(0)$ & \\
\hline
\end{tabular}

Data are presented as n (\%). BCG: Bacille Calmette-Guérin. TST: tuberculin skin test. QFN-G-IT: Quantiferon-TB Gold in tube.

Two-tailed statistical test was used to compare the qualitative results of TST, QFN-G-IT and T-SPOT.TB, respectively, between the two degree of exposure categories in adults and children.

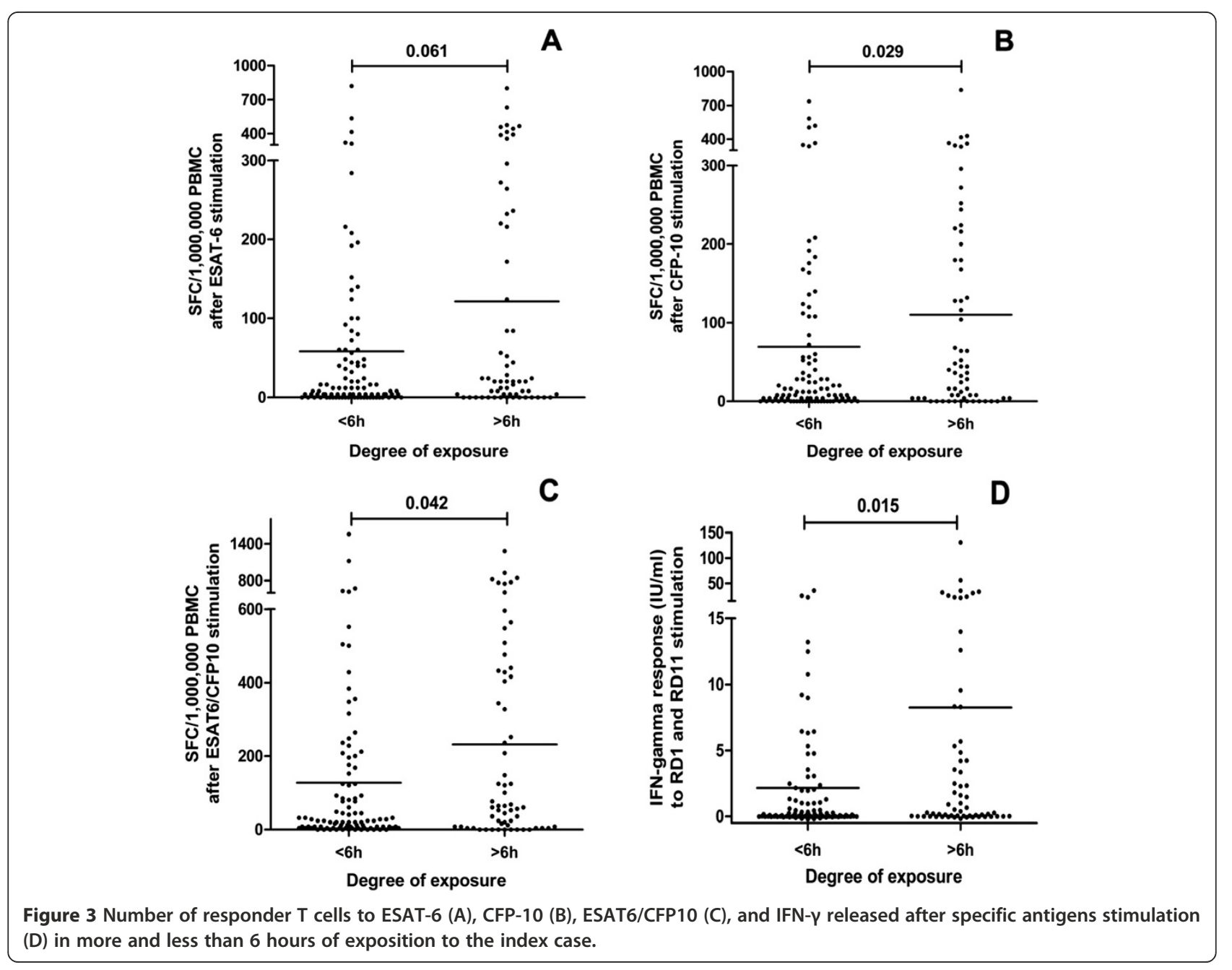


Table 5 Risk factors associated with the positivity of the TST, QFN-G-IT and T-SPOT.TB in adults

\begin{tabular}{|c|c|c|c|c|c|c|c|c|c|}
\hline \multirow{3}{*}{$\begin{array}{l}\text { Risk } \\
\text { factors }\end{array}$} & \multicolumn{3}{|c|}{ TST } & \multicolumn{3}{|c|}{ QFN-G-IT } & \multicolumn{3}{|c|}{ T-SPOT.TB } \\
\hline & \multicolumn{3}{|c|}{ n (\%) } & \multicolumn{3}{|c|}{ n (\%) } & \multicolumn{3}{|c|}{ n (\%) } \\
\hline & Positive & Negative & $p$ & Positive & Negative & $p$ & Positive & Negative & $p$ \\
\hline \multicolumn{10}{|l|}{ Cough } \\
\hline Yes & 34 (94.4) & $2(5.6)$ & 0.929 & $22(61.1)$ & $14(38.9)$ & 0.001 & $25(69.5)$ & $11(30.5)$ & 0.007 \\
\hline Not & 31 (93.9) & $2(6.1)$ & & $7(21.2)$ & $26(78.8)$ & & $12(36.4)$ & 21 (63.6) & \\
\hline \multicolumn{10}{|c|}{ Diagnostic delay } \\
\hline$<30$ & $35(97.2)$ & $1(2.8)$ & 0.244 & $11(30.5)$ & $25(69.5)$ & 0.565 & $14(38.9)$ & $22(61.1)$ & 0.077 \\
\hline $30-59$ & $13(76.5)$ & $4(23.5)$ & & $7(41.2)$ & $10(58.8)$ & & $7(41.2)$ & $10(58.8)$ & \\
\hline $60-89$ & $17(85)$ & $3(15)$ & & $7(35)$ & $13(65)$ & & $9(45)$ & $11(55)$ & \\
\hline$>89$ & $25(100)$ & $0(0)$ & & $12(48)$ & $13(52)$ & & $18(72)$ & $7(28)$ & \\
\hline \multicolumn{10}{|c|}{ Room's size $\left(m^{2}\right)$} \\
\hline$\leq 50$ & $2(66.7)$ & $1(33.3)$ & 0.462 & $1(33.3)$ & $2(66.7)$ & 0.020 & $2(66.7)$ & $1(33.3)$ & 0.023 \\
\hline $51-79$ & 15 (78.9) & $4(21.1)$ & & $4(21.1)$ & 15 (78.9) & & $6(31.6)$ & $13(68.4)$ & \\
\hline$\geq 80$ & $19(90.5)$ & $2(9.5)$ & & $14(66.7)$ & $7(33.3)$ & & $16(76.2)$ & $5(23.8)$ & \\
\hline \multicolumn{10}{|c|}{ Overcrowding } \\
\hline$\leq 15$ & 7 (87.5) & $1(12.5)$ & 0.800 & $2(28.6)$ & $5(71.4)$ & 0.480 & $5(62.5)$ & $3(37.5)$ & 0.820 \\
\hline$>15$ & 26 (83.9) & $5(16.1)$ & & 16 (51.6) & $15(48.4)$ & & $18(58.1)$ & $13(41.9)$ & \\
\hline
\end{tabular}

TST: tuberculin skin test. QFN-G-IT: Quantiferon-TB Gold in tube.

Diagnostic delay: days between first symptoms and TB diagnostic. Overcrowding: floor area index per person (square meters per person).

Two-tailed statistical test was used to compare the qualitative results of TST, QFN-G-IT and T-SPOT.TB, respectively, between the different categories of the risk

factors studied.

cough and with the household size, but in this case, inversely to what was expected: we found more positive results, the more square meters the room contained. In T-SPOT.TB results, there was a trend towards an association with diagnostic delay. Overcrowding was not associated with the positivity of any of the tests. The unexpected result of the household size was attributed to the association found between household size and the degree of exposure: contacts living in larger homes had been exposed longer to the index case. Logistic regression analysis was not performed since the number of cases having all the variables to be analyzed, was insufficient.

\section{Discussion}

To our knowledge, this is the first study that has investigated the role of the environment and of host-related risk factors for TB infection using TST and IGRAs. We compared the risk of positivity of TST, QFN-GIT and T-SPOT.TB and related them to risk factors of the index case, such as presence of a cough and delayed diagnosis, and also included environmental characteristics, such as household size and overcrowding.

The use of IGRAs has increased specificity in the diagnosis of LTBI, and has proven greater cost efficiency, especially in the use of preventive therapy $[15,16]$. Several studies have compared the performance of the IGRAs against the performance of TST, taking into account the time of exposure from a contagious source and the infectiousness of the source [17-19].

In our experience, the agreement between IGRA tests and TST was slight (especially in BCG-vaccinated), while the agreement between both IGRA tests was substantial and similar in BCG-unvaccinated and BCG-vaccinated contacts. The results suggest, as in previous studies, that IGRA tests are less affected by BCG vaccination than TST $[9,19]$.

In general there is a recognized low capacity of IGRAs to detect LTBI in children, especially in young children. In our study, it is important to point out that the percentage of TST-positive results (in children and adults) was high because most were referred to the Unit for study by positive TST after a contact. This may explain the high percentage of positive results obtained in children included in the study.

A possible explanation for the lack of relation observed between the positivity of the tests and the degree of exposure, could be the fact that we enrolled only contacts of patients showing smear-positive TB. This may have biased the sample of patients towards being more advanced in the disease, thus underestimating the effects of other variables. This inclusion criteria, was used in order to increase the likelihood of positive TST, attempting to get the majority of cases due to recent contact with the case study and not simply due to a previous infection. 
The number of responder $\mathrm{T}$ cells and the amount of IFN- $\gamma$ released could be a better indicator of infectiousness, than the qualitative positivity of an IGRA test, as seen in other studies [20]. We even found correlation between the degree of exposure and the number of responder $\mathrm{T}$ cells to CFP-10 and ESAT6/CFP10 by TSPOT.TB and the amount of IFN- $\gamma$ released in adults.

Most transmissions occur between the onset of coughing and the starting of the treatment [21]. It is known that the delay in diagnosis determines disease progression, increasing affected lung areas with the occurrence of cavitated lesions, extending the probability of infecting a new contact. In this study, neither TST nor IGRAs demonstrated to be related to diagnostic delay.

Sputum smear positivity should be considered a risk factor for infectiousness but there are both epidemiologic [22] and experimental studies [23] that have shown considerable variability of infectiousness among sputum smear-positive patients. Some studies suggested that the presence of a cough could generate potentially infectious aerosols that may increase the risk of disease transmission, regardless of the sputum smear status in the index case $[24,25]$. Our results showed that the positivity of both IGRA tests was associated with the presence of a cough. The potential of infectiousness is related to the patient's ability to aerosolise bacilli and to the number of bacilli that are aerosolised. In a study in Uganda, contacts of $\mathrm{TB}$ patients who produced high aerosols were more likely to have a new infection, as opposed to those who produced low aerosols, and the aerosol negative cases [26].

Since we enrolled only contacts with smear positive TB patients, it was not possible to examine the effect of smear negative and the positivity of tests when the index case had a cough. Guwatudde et al. [27], using TST, studied 1,206 household contacts with smear positive pulmonary. All the TB index cases had a cough for more than 3 weeks and $86 \%$ had an advanced disease. Among the host-related risk factors (including overcrowding), they found that only cavitary disease and a prolonged contact with an index case were independently associated with TB in the contact, and the "muzigo" type of housing; a building with multiple rooms that often share air space.

These findings are similar to those of our study, where we did not find a correlation between the overcrowding with the positivity of the tests. The number of patients living in crowded conditions was low in our sample, probably due to the characteristics of the individuals: almost 50\% natives and 50\% immigrants from Latin American countries, a kind of population with enough resources to live in family groups, in relatively good living conditions. The other type of population was from the community group, usually people sharing the same air for a full working day. In our study, we found a reverse correlation between the size of the room and the positivity of the IGRAs, but associated this correlation with a higher degree of exposure.

Although data about ventilation was not collected in this study, it suggests that in relation to environmental risk factors, more than the number of people in contact with the TB case in a limited environment, it is the ventilation of the room that is the main determinant in the transmission of infection in addition to the time of exposure. A study carried out in Peru found that opening windows and doors provided more than double the amount of ventilation of the mechanical ones and the risk of TB infection was lower [28].

The main limitation of our study was the small number of patients included, given the difficulty of enrolling patients with smear-positive pulmonary TB in our environment. However, the results are sufficiently consistent to show the strong association between the IGRAs and the risk factors involved in the LTBI.

\section{Conclusions}

In conclusion, comparing the performance of the TST and IGRA tests in contacts with smear positive pulmonary TB, we found that both IGRAs were associated with certain host-related risk factors involved in the transmission of disease, such as the presence of cough. On the other hand, the study suggests that some environmental risk factors, such as the lack of ventilation associated with the time of exposure, may be crucial for the transmission, aside from the status smear of the index case and the diagnostic delay. A larger study would be needed, in order to confirm all the findings and better characterize the true role of the different variables involved in the transmission of the disease.

\section{Abbreviations}

TST: Tuberculin skin test; IGRA: Interferon-gamma released assays HIV, Human immunodeficiency virus; TNF: Tumor necrosis factor; QFN-G-IT: Quantiferon-TB Gold in tube; LTBI: Latent tuberculosis infection.

\section{Competing interests}

None of the investigators have relevant financial interest in or a competing interest with the subject matter or materials discussed in this manuscript. None of the Scientific Societies, neither QIAGEN nor Oxford Immunotec (Abingdon, UK) had a hole in the study, conduct, collection, management, analysis, or interpretation of the data, or preparation, review, or approval of the manuscript.

\section{Authors' contributions}

MLSG, IL and NAG conceived and designed the study. MLSG, NAG, CM MAJF, JS, MAS, AC and JRM contributed to acquisition and analysis of data from hospital wards, and IL and JD to acquisition and analysis of data in the laboratory. MLSG, IL and JD wrote the manuscript with significant contributions from NAG, MAJF, CM, JS, MAS, AC and JRM. All authors read and approved the final manuscript.

\section{Authors' information}

Maria Luiza de Souza-Galvão: MD, Irene Latorre: MSc, Ph.D, Neus AltetGómez: MD, Ph.D, Celia Milá MD, María Ángeles Jiménez MD, Jordi Solsona MD, Maria Asunción Seminario MD, Adela Cantos BSc, Juan Ruiz-Manzano: MD, Ph.D, and José Domínguez MSc, PhD. 
The authors are members of the European Tuberculosis Network (TB-NET) Group.

J. Dominguez is a researcher funded from the Miguel Servet programme of the Instituto de Salud Carlos III (Spain).

Irene Latorre is currently a research from ICREA Infection Biology Laboratory, Department of Experimental and Health Sciences, Universitat Pompeu Fabra Barcelona, (Spain).

\section{Acknowledgments}

We thank the nursing staff of the Unitat de Tuberculosi de Drassanes Barcelona; J.J. Zamora and I. Casas for technical assistance in managing database and statistical analyses; also A. Rodés, J. Caylà and A Orcau for the epidemiological data provided; P. Bokor for assistance with English manuscript edition.

\section{Funding}

This work was supported by a grant from the Fundación IDIAP Jordi Gol; the Sociedad Española de Neumología y Cirugía Torácica (SEPAR); the Societat Catalana de Pneumologia (SOCAP); the Fundació Catalana de Pneumologia (FUCAP) and the Spanish Health Research Fund (FIS, PI 10/00214).

\section{Author details}

${ }^{1}$ Unitat de Tuberculosi de Drassanes, Hospital Universitari Vall d'Hebron, Barcelona, Spain. ${ }^{2}$ IDIAP Jordi Gol Research Foundation, Barcelona, Spain. ${ }^{3}$ Universitat Autònoma of Barcelona, Barcelona, Spain. ${ }^{4}$ Servei de Microbiologia, Institut d'Investigació Germans Trias i Pujol, Badalona, Spain. ${ }^{5}$ Servei de Pneumologia, Hospital Universitari Germans Trias i Pujol, Badalona, Spain. ${ }^{6}$ CIBER Enfermedades Respiratorias, Madrid, Spain.

Received: 25 February 2014 Accepted: 9 May 2014

Published: 13 May 2014

\section{References}

1. World Health Organization: Global tuberculosis report 2013 (WHO/HTM/ TB/2013.11) Geneva, Switzerland. 2013, http://www.who.int/tb/ publications/global_report/en/.

2. Styblo K: Recent advances in epidemiological research in tuberculosis. Adv Tuberc Res 1980, 20:1-63.

3. Borrell S, Español M, Orcau A, Tudó G, March F, Caylà JA, Jansà JM, Alcaide F, Martín-Casabona N, Salvadó M, Martínez JA, Vidal R, Sánchez F, Altet N, Coll P, González-Martín J: Factors associated with differences between conventional contact tracing and molecular epidemiology in study of tuberculosis transmission and analysis in the city of Barcelona, Spain. J Clin Microbiol 2009, 47:198-204.

4. Erkens CG, Kamphorst M, Abubakar I, Bothamley GH, Chemtob D, Haas W, Migliori GB, Rieder HL, Zellweger JP, Lange C: Tuberculosis contact investigation in low prevalence countries: a European consensus. Eur Respir J 2010, 36:925-949.

5. Diel R, Goletti D, Ferrara G, Bothamley G, Cirillo D, Kampmann B, Lange C, Losi M, Markova R, Migliori GB, Nienhaus A, Ruhwald M, Wagner D, Zellweger JP, Huitric E, Sandgren A, Manissero D: Interferon-gamma release assays for the diagnosis of latent mycobacterium tuberculosis infection: a systematic review and meta-analysis. Eur Respir J 2011, 37:88-99.

6. Beggs CB, Noakes CJ, Sleigh PA, Fletcher LA, Siddiqi K: The transmission of tuberculosis in confined spaces: an analytical review of alternative epidemiological models. Int J Tuberc Lung Dis 2003, 7:1015-1026.

7. Tornee S, Kaewkungwal J, Fungladda W, Silachamroon U, Akarasewi P, Sunakorn P: The association between environmental factors and tuberculosis infection among household contacts. Southeast Asian J Trop Med Public Health 2005, 36(Suppl 4):221-224

8. Altet Gómez MN, Alcaide Megías J, Canela Soler J, Milá Augé C, Jiménez Fuentes MA, de Souza Galvao ML, Solsona Peiró J: Pulmonary symptomatic tuberculosis' diagnostic delay study. Arch Bronconeumol 2003, 39:146-152.

9. Domínquez J, Ruiz-Manzano J, De Souza-Galvão M, Latorre I, Milà C, Blanco S, Jiménez MA, Prat C, Lacoma A, Altet N, Ausina V: Comparison of two commercially available gamma interferon blood tests for immunodiagnosis of tuberculosis. Clin Vaccine Immunol 2008, 15:168-171.

10. Diel R, Loddenkemper R, Meywald-Walter K, Gottschalk R, Nienhaus A: Comparative performance of tuberculin skin test, QuantiFERON-TB-gold in tube assay, and T-spot.TB test in contact investigations for tuberculosis. Chest 2009, 135:1010-1018.

11. Domínguez J, De Souza-Galvão M, Ruiz-Manzano J, Latorre I, Prat C, Lacoma A, Milà C, Jiménez MA, Blanco S, Maldonado J, Altet N, Ausina V: T-cell responses to the Mycobacterium tuberculosis-specific antigens in active tuberculosis patients at the beginning, during, and after antituberculosis treatment. Diagn Microbiol Infect Dis 2009, 63:43-51.

12. UN Department of Economic and Social Affairs. Division for Sustainable Development: CSD theme indicator framework from 2001. http://www.un. org/esa/sustdev/csd/csd9_indi_bp3.pdf.

13. Ruiz-Manzano J, Blanquer R, Calpe JL, Caminero JA, Caylà J, Domínguez JA, García JM, Vidal R, Spanish Society of Pulmonology and Thoracic Surgery: Diagnosis and treatment of tuberculosis. Arch Bronconeumol 2008, 44:551-566.

14. González-Martín J, García-García JM, Anibarro L, Vidal R, Esteban J, Blanquer R, Moreno S, Ruiz-Manzano J: Consensus document on the diagnosis, treatment and prevention of tuberculosis. Arch Bronconeumol 2010, 46:255-274.

15. Latorre I, De Souza-Galvão M, Ruiz-Manzano J, Lacoma A, Prat C, Altet N, Ausina V, Domínguez J: Evaluating the non-tuberculous mycobacteria effect in the tuberculosis infection diagnosis. Eur Respir J 2010, 35:338-342.

16. Nienhaus A, Schablon A, Costa JT, Diel R: Systematic review of cost and cost-effectiveness of different TB-screening strategies. BMC Health Serv Res 2011, 11:247.

17. Shams H, Weis SE, Klucar P, Lalvani A, Moonan PK, Pogoda JM, Ewer K, Barnes PF: Enzyme-linked immunospot and tuberculin skin testing to detect latent tuberculosis infection. Am J Respir Crit Care Med 2005, 172:1161-1168,

18. Lalvani A, Pathan AA, Durkan H, Wilkinson KA, Whelan A, Deeks JJ, Reece WH, Latif M, Pasvol G, Hill AV: Enhanced contact tracing and spatial tracking of Mycobacterium tuberculosis infection by enumeration of antigen-specific T cells. Lancet 2001, 357:2017-2021.

19. Altet-Gómez N, De Souza-Galvao M, Latorre I, Milà C, Jiménez MA, Solsona J, Cantos A, Zamora JJ, Ruiz-Manzano J, Ausina V, Domínguez J: Diagnosing TB infection in children: analysis of discordances using in vitro tests and the tuberculin skin test. Eur Respir J 2011, 37:1166-1174.

20. Latorre I, De Souza-Galvão M, Ruiz-Manzano J, Lacoma A, Prat C, Fuenzalida L, Altet N, Ausina V, Domínguez J: Quantitative evaluation of T-cell response after specific antigen stimulation in active and latent tuberculosis infection in adults and children. Diagn Microbiol Infect Dis 2009, 65:236-246.

21. Storla DG, Yimer S, Bjune GA: A systematic review of delay in the diagnosis and treatment of tuberculosis. BMC Public Health 2008, 8:15.

22. Brooks SM, Lassiter NL, Young EC: A pilot study concerning the infection risk of sputum positive tuberculosis patients on chemotherapy. Am Rev Respir Dis 1973, 108:799-804.

23. Sultan L, Nyka W, Mills C, O'Grady F, Wells W, Riley RL: Tuberculosis disseminators. A study of the variability of aerial infectivity of tuberculous patients. Am Rev Respir Dis 1960, 82:358-369.

24. Fennelly KP, Martyny JW, Fulton KE, Orme IM, Cave DM, Heifets LB: Coughgenerated aerosols of mycobacterium tuberculosis: a new method to study infectiousness. Am J Respir Crit Care Med 2004, 169:604-609.

25. Fennelly KP, Jones-López EC, Ayakaka I, Kim S, Menyha H, Kirenga B, Muchwa C, Joloba M, Dryden-Peterson S, Reilly N, Okwera A, Elliott AM, Smith PG, Mugerwa RD, Eisenach KD, Ellner JJ: Variability of infectious aerosols produced during coughing by patients with pulmonary tuberculosis. Am J Respir Crit Care Med 2012, 186:450-457.

26. Jones-López EC, Namugga O, Mumbowa F, Ssebidandi M, Mbabazi O, Moine S, Mboowa G, Fox MP, Reilly N, Ayakaka I, Kim S, Okwera A, Joloba M, Fennelly KP: Cough aerosols of mycobacterium tuberculosis predict new infection: a household contact study. Am J Respir Crit Care Med 2013, 187:1007-1015.

27. Guwatudde D, Nakakeeto M, Jones-Lopez EC, Maganda A, Chiunda A Mugerwa RD, Ellner JJ, Bukenya G, Whalen CC: Tuberculosis in household contacts of infectious cases in Kampala, Uganda. Am J Epidemiol 2003, 158:887-898.

28. Escombe AR, Oeser CC, Gilman RH, Navincopa M, Ticona E, Pan W, Martínez C, Chacaltana J, Rodríguez R, Moore DA, Friedland JS, Evans CA: Natural ventilation for the prevention of airborne contagion. PLOS Med 2007, 4:e68.

\section{doi:10.1186/1471-2334-14-258}

Cite this article as: de Souza-Galvão et al:: Correlation between tuberculin skin test and IGRAs with risk factors for the spread of infection in close contacts with sputum smear positive in pulmonary tuberculosis. BMC Infectious Diseases 2014 14:258. 